\title{
New Line-Interactive UPS System with DSP-based Active Power-Line Conditioning
}

\author{
R. Cheung, L. Cheng, P. Yu \\ Ryerson Polytechnic University \\ Department of Electrical Engineering \\ 350 Victoria Street, Toronto, Ontario \\ Canada M5B 2K3
}

\author{
R. Sotudeh \\ University of Teesside \\ School of Science \& Technology \\ Middlesbrough, Cleveland \\ UK TS1 3BA
}

\begin{abstract}
This paper presents a new line-interactive UPS (uninterruptible power supply) system with active power-line conditioning capability. This system uses one power electronic inverter to implement two functions: maintaining uninterruptible power for critical loads, and suppressing power-line harmonics. The system offers efficient and economical power conditioning. Experimental validation is given.
\end{abstract}

\section{Introduction}

Critical loads such as computers and process equipment are vulnerable to power disturbances or interruptions. On the other hand, many of these loads use switching devices and may generate considerable harmonic disturbances which can create problems for neighbouring sensitive electronic equipment. Uninterruptible power supplies (UPS) can be used to protect critical loads against power interruptions. However, UPS themselves may inject additional harmonic disturbances on the system side, since they use switching devices and are designed primarily to protect the load but not the system. Power conditioners such as line regulators, passive filters, and active filters can be used to suppress powerline harmonic disturbances [1]. This paper presents a new line-interactive UPS system with active power-line conditioning capability. This new system uses one simple power electronic inverter to implement two functions: maintaining uninterruptible power for critical loads, and suppressing power-line harmonic disturbances. A 10-kVA inverter system was built to provide experimental validation.

\section{Standard UPS Systems}

Today the UPS technology is quite mature. There are two standard UPS topologies [2]:

(1) Off-line UPS contains a rectifier, a battery, an inverter, and a transfer switch. The normal flow of power is directly from the supply to the load through the transfer switch. In the event of supply outages, the switch transfers the load to the inverter which operates from the battery. Due to the requirement of transfer operations, the UPS does not provide a complete disturbance isolation and power protection for critical loads.

(2) On-line UPS is similar to the off-line one, but the transfer switch is normally connected to the inverter. The normal power flow is from the supply through the rectifier and the inverter to the load. In the event of supply failure, the power flows from the battery through the inverter to the load. There is normally no transfer operation except when the UPS needs maintenance. This UPS continuously conditions its output to the load. Therefore it provides better protection than the offline UPS. It is however more expensive and usually larger than the off-line one.

\section{Standard Active Power-Line Conditioners}

Active power-line conditioners basically consist of a power electronic inverter which monitors the current waveform distortion in the power line and inject appropriate current to cancel the distortion [3]. Unlike UPS primarily for load protection, the standard active conditioner is designed to suppress 
power-line harmonic disturbances on the system side.

\section{New Line-Interactive UPS with Active Power-Line Conditioning Capability}

This paper proposes a simple power electronic circuit which combines the features of standard online UPS, off-line UPS, and active power-line conditioner. The circuit shown in Figure 1 uses one inverter to implement two functions: maintaining uninterruptible power for critical loads, and suppressing power-line harmonic disturbances.

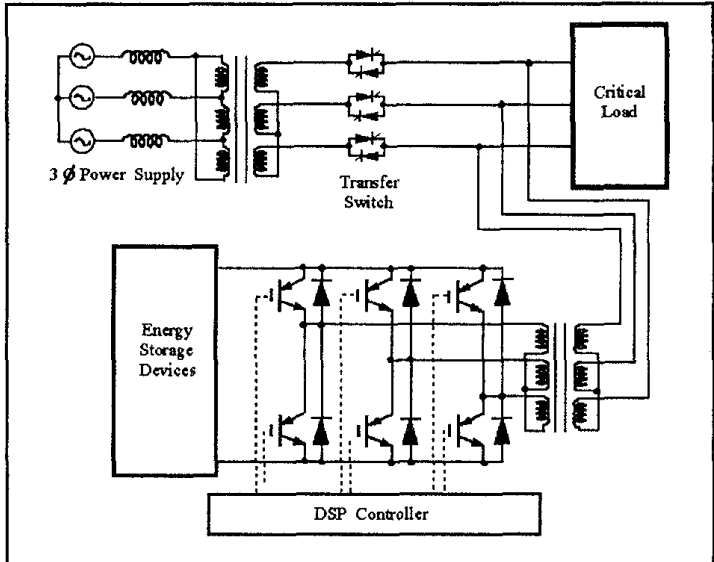

Figure 1 Basic System Configuration of the New LineInteractive UPS System

The corresponding operations are:

(1) Line-interactive UPS operation - Like off-line UPS, the normal flow of power in the proposed circuit is directly from the supply to the load through the static switch. Upon a supply failure the direct flow of power from the utility is interrupted, and the inverter immediately delivers power from the battery to the load. Like on-line UPS, this operation requires no power transfer and does not cause any power interruption. The inverter, shunt-connected with the load, operates interactively with the system. This is also known as line-interactive UPS operation.

(2) Active power-line conditioning operation During the normal flow of power, the inverter operates as an active power-line conditioner and eliminates any harmonics injected from non-linear loads into the supply system.

\section{Simple Circuit with Advanced DSP Control}

Figure 1 shows a simple circuit for the proposed line-interactive UPS. The circuit consists of a voltage-source inverter, a battery, a transformer, and a switch. This circuit is similar to a standard circuit for active power-line conditioners with the exception that the energy storage uses a battery, instead of a capacitor for standard active conditioners. The battery not only provides energy for critical loads to ride through the period of power interruptions, but also enhances the circuit's power-line conditioning capability. The standard active conditioner often faces energy problems for compensating longer term power-line disturbances such as voltage sages. The circuit uses only one inverter to implement two functions: UPS and active power conditioning. This may require three power electronic converters in standard circuits: two for UPS (rectifier and inverter) and one for conditioner (inverter).

The circuit uses an advanced DSP (digital signal processor) control for power-line conditioning operation. The following shows typical control formulations using a new modulation method which improves implementation and controllability of a popular instantaneous power method [4].

(1) Transform power-line three-phase currents into $\alpha \beta$ coordinates.

$$
\left[\begin{array}{l}
I_{\alpha} \\
I_{\beta}
\end{array}\right]=\sqrt{\frac{2}{3}}\left[\begin{array}{ccc}
1 & \frac{-1}{2} & \frac{-1}{2} \\
0 & \frac{\sqrt{3}}{2} & \frac{-\sqrt{3}}{2}
\end{array}\right]\left[\begin{array}{l}
I_{a} \\
I_{b} \\
I_{c}
\end{array}\right]
$$

(2) Perform a sinusoidal transformation of the $\alpha \beta$ currents.

$$
\left[\begin{array}{c}
\mathrm{Ip} \\
\mathrm{Iq}
\end{array}\right]=\left[\begin{array}{cc}
\mathrm{m}_{\mathrm{S}} & \mathrm{m}_{\mathrm{C}} \\
-\mathrm{m}_{\mathrm{C}} & \mathrm{m}_{\mathrm{S}}
\end{array}\right]\left[\begin{array}{l}
\mathbf{i}_{\alpha} \\
\mathbf{i}_{\beta}
\end{array}\right]
$$

where $\mathrm{m}_{\mathrm{s}}=\sin \omega \mathrm{t}$ and $\mathrm{m}_{\mathrm{c}}=-\cos \omega \mathrm{t}$. $\mathrm{i}_{\mathrm{p}}$ and $\mathrm{i}_{\mathrm{q}}$ contain dc and ac components. 
(3) Eliminate the dc component, and inversely transform the ac component to $\alpha \beta$ currents.

$$
\left[\begin{array}{l}
\mathbf{l}_{\alpha} \\
\mathbf{I}_{\beta}
\end{array}\right]=\left[\begin{array}{cc}
\mathbf{m}_{\alpha} & -\mathbf{m}_{\beta} \\
\mathbf{m}_{\beta} & \mathbf{m}_{\alpha}
\end{array}\right]\left[\begin{array}{l}
\mathbf{I} \mathbf{p} \\
\mathbf{I q}
\end{array}\right]
$$

(4) Obtain the control reference for the power-line conditioner from above $\alpha \beta$ currents.

$$
\left[\begin{array}{l}
\mathrm{Ia}^{*} \\
\mathrm{Ib}^{*} \\
\mathrm{Ic}^{*}
\end{array}\right]=\sqrt{\frac{3}{2}}\left[\begin{array}{cc}
1 & 0 \\
-\frac{1}{2} & \frac{\sqrt{3}}{2} \\
-\frac{1}{2} & -\frac{\sqrt{3}}{2}
\end{array}\right]\left[\begin{array}{l}
\mathrm{Ih}_{\alpha} \\
\mathrm{Ih}_{\beta}
\end{array}\right]
$$

\section{Simulation}

The simulation is used to demonstrate efficiency of the new line-interactive UPS system. The system has three basic power conversion operations: 1) power-line harmonics suppression, 2) energy storage charging, and 3) UPS power conversion. Figure 1 shows the system configuration used in the simulations.

\section{Power-Line Harmonics Suppression}

Under the normal power-line conditions, the new line-interactive UPS system acts like a power-line conditioner and is capable of suppressing harmonics generated by non-linear loads connected on the power line. A non-linear load having a three-phase rectifier at the input stage is used to demonstrate the capability of the system. Figure 2a shows the distorted power line current due to the non-linear load. The system monitors the power-line distortions and injects equal-butopposite distortions into the power line to cancel the original ones. The performance of the system is simulated. Figure $2 \mathrm{~b}$ shows the simulated compensating current generated by the system. Figure $2 \mathrm{c}$ shows the power-line current after the distorted load current has been compensated by the system. As shown in the figure, all the current harmonics in the power line are suppressed.

\section{Battery Charging}

The battery in the new line-interactive UPS system
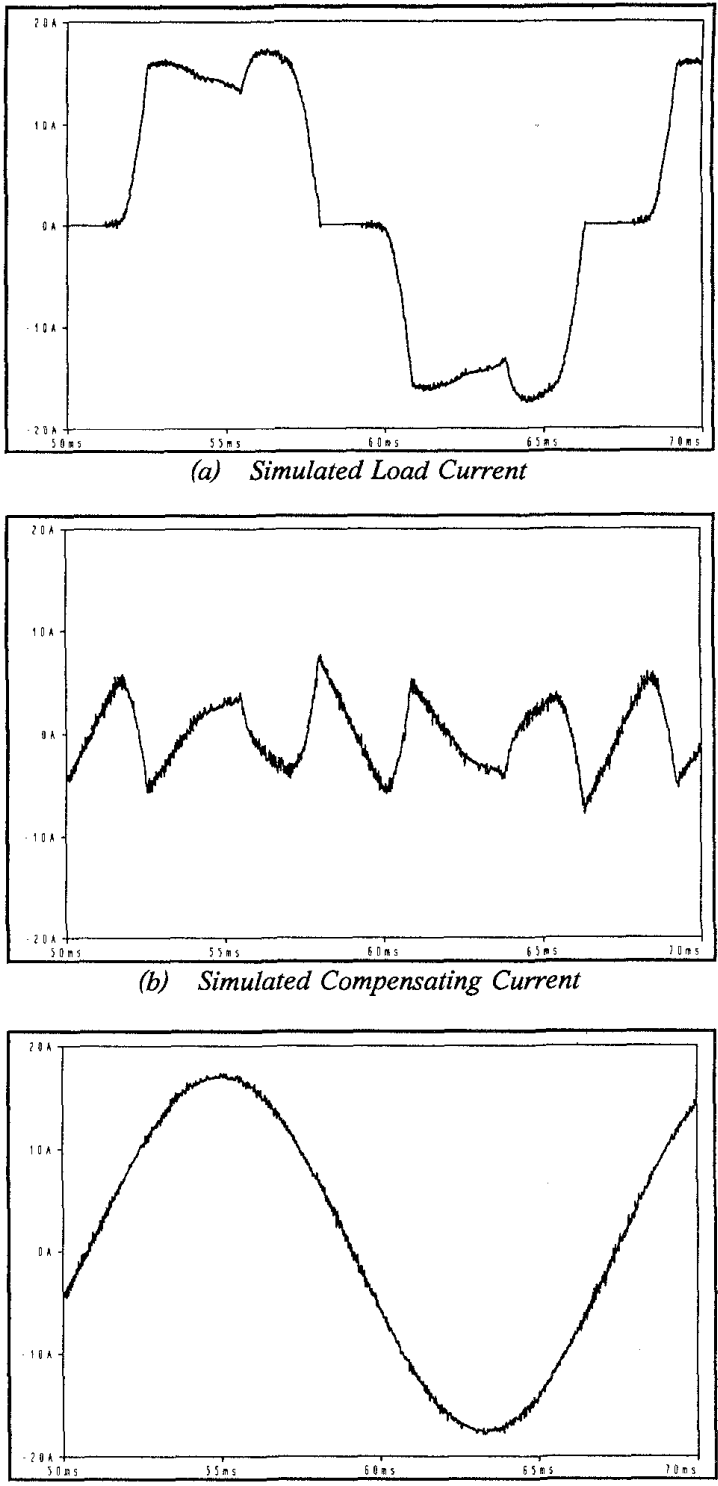

(c) Simulated Compensated Line Current

Figure 2 Simulated Active Power-Line Conditioning

is used to supply power to critical loads during a power interruption. The battery condition is continuously monitored, and the battery is charged whenever its voltage drops below a preset threshold during the normal power condition. Figure $3 \mathrm{a}$ shows the simulated input current of the new line-interactive UPS system when the system is conducting simultaneously two operations: 1 ) compensating the distortion load current, and 2) 
charging the battery through the same inverter. Figure $3 \mathrm{~b}$ shows the compensated power-line current.

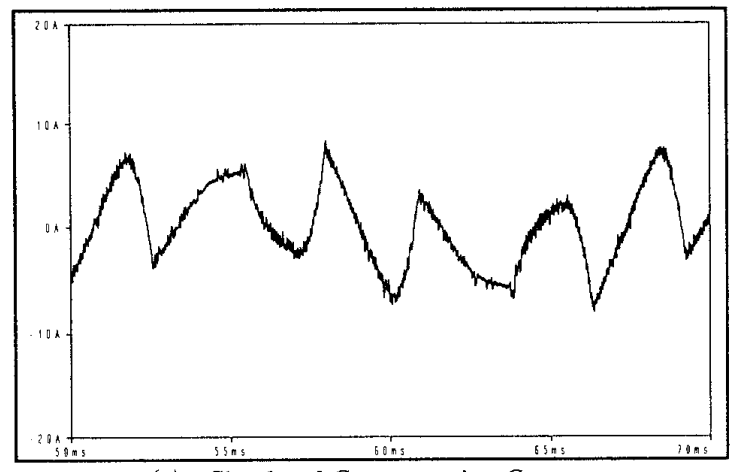

(a) Simulated Compensating Current

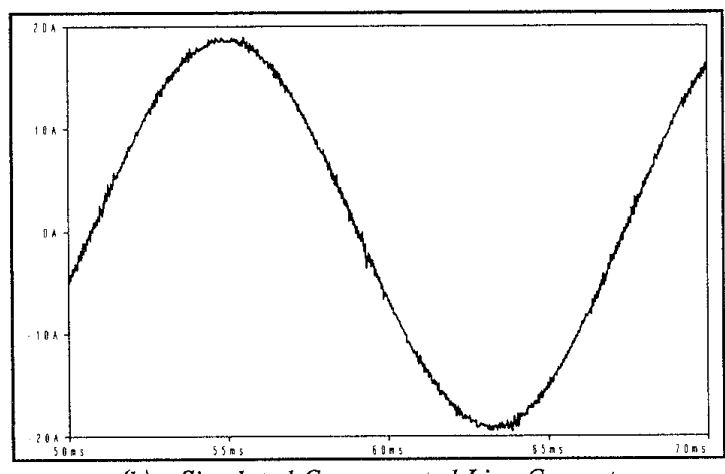

(b) Simulated Compensated Line Current

Figure 3 Simulated Battery Charging Operation

\section{Power Interruption}

During the power interruption, the new lineinteractive UPS is used to supply continuous power to the critical loads. Figure 4a shows the line voltage waveform, and Figure $4 \mathrm{~b}$ gives the line current waveform.

\section{Experimental Validation}

Recently, a prototype of a 10-kVA line-interactive UPS using the new DSP control has been built and tested. The basic circuit of the prototype is shown in Figure 1. The main component of the prototype is a simple voltage-source power electronic inverter which uses a battery as energy storage and transformer as current choke. Figure 5 shows the measurements on the power line under a normal
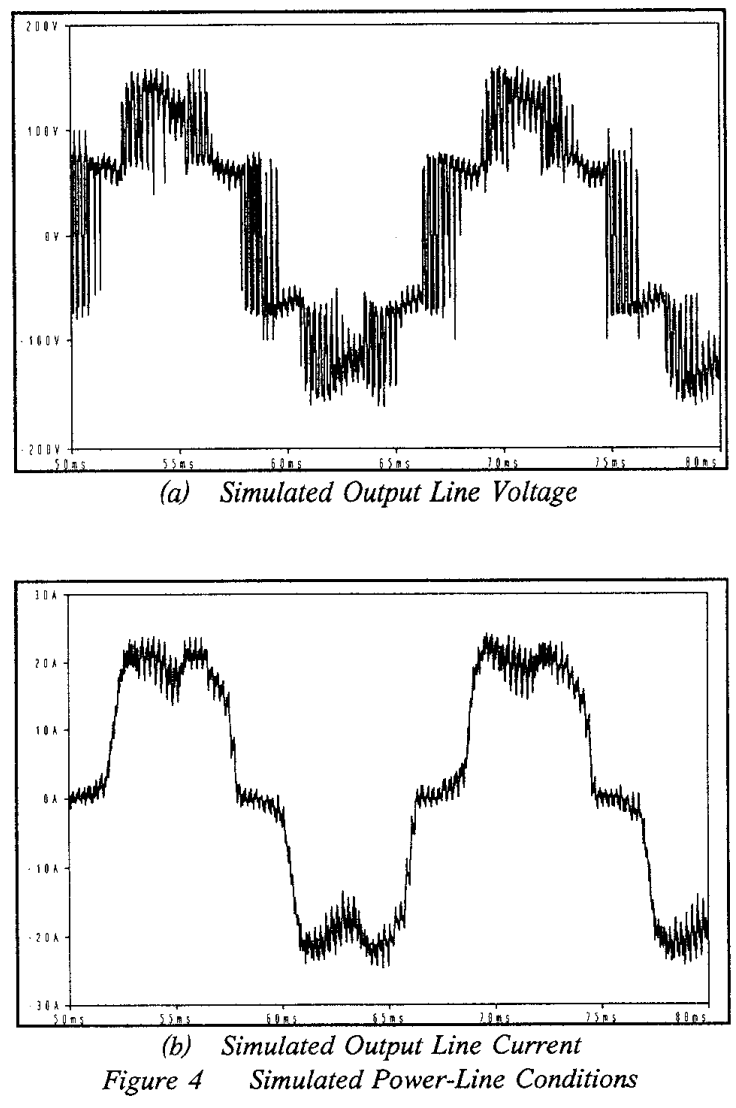

condition that the line-interactive UPS operates as an active power-line conditioner. The current harmonics injected by a critical load is suppressed from a level of $26 \%$ to a level of less than $4 \%$. Figure 6 shows the measurements during a power interruption that the line-interactive UPS provides continuous power to the load.

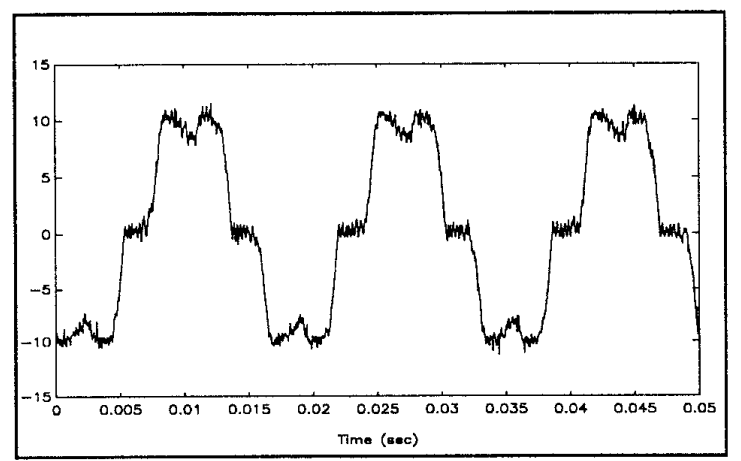

(a) Measured Load Current 


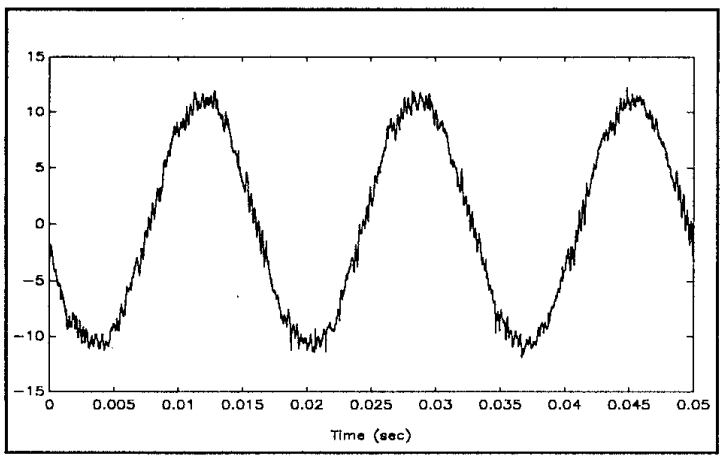

(b) Measured Compensated Line Current

Figure 6 Active Power-Line Conditioning Operation

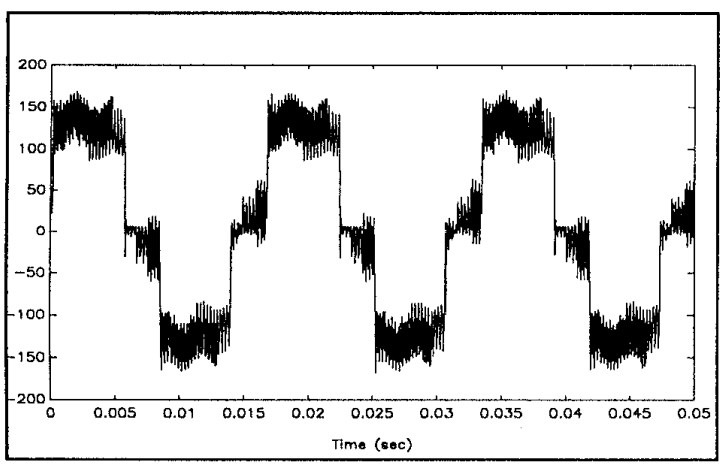

(a) Measured Inverter Output Voltage

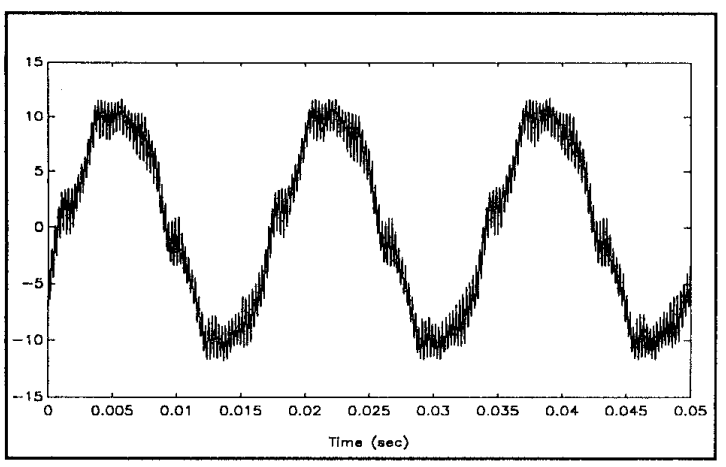

(b) Measured Inverter Output Current

Figure 6 UPS Operation during Power Interruption

\section{Conclusion}

This paper present a new line-interactive UPS system with active power-line conditioning capability. Computer simulations and experimental results have demonstrated that the new system is capable of using one inverter to implement two functions: maintaining uninterruptible power for critical loads, and suppressing power-line harmonics. The system offers an efficient and economical power conditioning for critical loads.

\section{References}

[1] H. Akagi, "Trends in Active Power Line Conditioners,", IEEE Power Elec., pp. 263-268, May 1994

[2] A. Kawamura, R. Chuarayapratip, T. Haneyoshi, "Deadbeat Control of PWM Inverter with Modified Pulse Patterns for Uninterruptible Power Supply,", IEEE Tran. Ind. Elec, Vol, 35, No. 2, pp. 295300, May 1988

[3] L. Gyugyi, E.C. Strycula, "Active AC Power Filters," IEEE Trans. on Ind. App., pp. 529-535, 1976

[4] L. Cheng, R. Cheung, R. Sotudeh, "Instantaneous Harmonic Current Compensation Using a Novel Modulation Scheme," Proc. of 29th UPEC, Vol. 1, pp. 121-124, 1994 